\title{
Optimal R\&D subsidies in a model with physical capital, human capital and varieties
}

\author{
Manuel A. Gómez ${ }^{\mathrm{a}, 1}$, Tiago N. Sequeira ${ }^{\mathrm{b}, 2,}$ \\ ${ }^{a}$ Universidad de A Coruña \\ ${ }^{b}$ Universidade da Beira Interior and CEFAGE-UBI research unit
}

\begin{abstract}
In this paper, we analyze the social planner solution of an endogenous growth model with physical capital, human capital and R\&D. The model incorporates three sources of inefficiency: monopolistic competition in the intermediate-goods sector, duplication externalities and spillovers in R\&D. A complete stability analysis for the optimal growth problem of this model is provided. We characterize the optimal policy that can decentralize the optimal solution and find that the path of the optimal R\&D subsidy can be non-monotonic.
\end{abstract}

JEL classification: H20, O40.

Keywords: Endogenous growth, R\&D, Optimal policy

\section{Introduction}

There is a broad consensus that physical capital accumulation, knowledge formation, and R\&Dbased technological progress are the three main engines of growth. For the most part, they have been considered as alternative rather than complementary explanations in the theoretical literature. As a notable exception, Arnold (2000a) and Funke and Strulik (2000) (AFS henceforth) proposed an integrated endogenous growth model with physical capital, human capital and R\&D, in which the economy passes through different stages of development. In the fully-industrialized phase three sectors are acting: the competitive final goods sector, the schooling sector where knowledge (human capital) is accumulated, and the intermediate-goods monopolistic sector which produces an increasing variety of goods due to $R \& D$.

However, monopoly power is not the only plausible source of inefficiency in R\&D-based growth models (see, e.g., the comprehensive review by Jones, 2005). Thus, empirical evidence reported, e.g., by Griliches (1992), Jones (1995), Engelbrecht (1997), del Barrio-Castro et al. (2002), Pessoa (2005) and Porter and Stern (2000) also supports the existence of R\&D spillovers in innovation - a "standing on shoulders" or a "fishing-out" effect. Several authors have also pointed out that the R\&D activity may be subject to an external effect associated to the duplication and overlap of research effort —a "stepping on toes" effect (e.g., Jones, 1995; Stokey, 1995; Pessoa, 2005; Porter and Stern, 2000). Intuitively, the larger the number of people searching for ideas is, the more likely it is that duplication of research would occur. Evidence of duplicative research has also been found, e.g., by Kortum (1993) and Lambson and Phillips (2007). Both external effects — spillovers

\footnotetext{
Email addresses: mago@udc.es (Manuel A. Gómez), sequeira@ubi.pt (Tiago N. Sequeira)

${ }^{1}$ Departamento de Economía Aplicada II, Universidad de A Coruña, Campus de Elviña, 15071 A Coruña, Spain. Tel.:+34 981167000. Fax: +34981 167070 .

${ }^{2}$ Departamento de Gestão e Economia. Universidade da Beira Interior. Estrada do Sineiro. 6200-209 Covilhã. Portugal. Tel.: +351 275319700. Fax: +351 275329183.
} 
in R\&D and duplication externalities - are neglected in the AFS model, which assumes that innovation depends exclusively and linearly on human capital devoted to R\&D. Additionally, Gómez (2011b) has recently examined the ability of the simplest AFS model to describe the development process and concluded that it can hardly be reconciled with data. First, Gómez (2011b) notes that previously reported simulations with the AFS model made by Funke and Strulik (2000), Gómez (2005) and Iacopetta (2010) feature three main problems, namely, instability of the steady state, too fast convergence, and unrealistic highly oscillatory dynamics which are at odds with data. Thereafter, Gómez (2011b) performs a detailed sensitivity analysis of the (two) stable roots of the fully industrialized economy which shows that numerical simulations with the AFS model could hardly yield realistic transition paths for plausible parameter values.

According with the empirical evidence, Sequeira (2011), Gómez (2011a,b) and Iacopetta (2011) have incorporated R\&D spillovers and duplication externalities into the AFS model. This modification largely complicates the dynamics of the economy, which passes from being described by a third- to a fifth-order dynamical system. However, as Gómez (2011a,b) shows, this add-on to the basic AFS setup also increases significantly its ability to fit the observed data. While the previously cited works focused on analyzing the transition dynamics of the market economy and its fit to data, the incorporation of $R \& D$ spillovers and duplication externalities to the model raises the question of whether an adequate government intervention can provide the required incentives to correct these inefficiencies, so as to make the decentralized economy replicate the first-best solution attainable by a social planner. None of these previous contributions has analyzed this issue in this framework, so this paper seeks to fill this gap.

This paper characterizes a dynamically optimal fiscal policy capable of making the decentralized economy achieve the first-best optimum in an extended version of the AFS endogenous growth model with physical capital, human capital and R\&D. The model incorporates three sources of inefficiency: monopolistic competition in the intermediate-goods sector, duplication externalities and spillovers in R\&D. We first study the decentralized economy with government. Next, we characterize the social planner solution, and derive an optimal R\&D subsidy policy that can decentralize the Pareto-efficient solution. The optimal growth path can be decentralized by means of a subsidy to production of intermediate goods at a constant rate combined with a time-varying subsidy (or tax) to R\&D. We also perform a detailed (local) stability analysis of the first-best solution, and find that the time path of the optimal R\&D subsidy can be non-monotonic. With the notable exceptions of Arnold (2000b) and Eicher and Turnovsky (1999) — in quite different setups from the one presented here - the stability analysis issue has been ignored in most of the related literature (e.g., Jones and Williams, 2000; Steger, 2005; Jones, 2005; Grossmann et al., 2010), probably because of its complexity and also because of the emphasis put on the quantitative assessment of distortions on the steady state - disregarding the transitional phase. However, the analysis of long-run effects may be misleading if the steady state is unstable because in this case the economy would not converge to it (unless, of course, it already starts on it). One then may wonder whether this is a real possibility or not. So, we show in the Appendix that if we had assumed (more unrealistically) that there are no duplication externalities, the steady state of the socially planned economy would be instable. Thus, stability cannot be simply taken for granted.

This paper is mostly related to Arnold (2000b) and Grossmann et al. (2010), who also characterize analytically the optimal dynamic fiscal policy in R\&D-based endogenous growth models. However, they do not include human capital as an engine of growth. In particular, Arnold (2000b) studies the optimal combination of production and R\&D subsidies in the Romer (1990) model. This model has been criticised because of the implied counterfactual scale effects and, furthermore, it does not include duplication externalities. Grossmann et al. (2010) consider instead a semi-endogenous growth model á la Jones (1995), in which economic growth is driven solely by 
exogenous population growth and, furthermore, they do not study analytically the stability of the centrally planned economy. The different assumptions lead to significantly different results, especially regarding the behaviour of the optimal R\&D subsidy. In particular, we find that the optimal R\&D subsidy can display a non-monotonic behaviour, which is in sharp contrast with the analytical results in Arnold (2000b) and the numerical results in Grossmann et al. (2010). Other related research (e.g., Jones and Williams, 2000; Alvarez-Pelaez and Groth, 2005; Steger, 2005; Strulik, 2007) has focused instead on the quantitative assessment of distortions - mainly on the long-run, and thus disregarding the transitional phase - by resorting to numerical simulations. Hence, the optimal fiscal policy is not characterized analytically.

The remaining of this paper is organized as follows. Section 2 describes the market economy. Section 3 analyzes the centralized economy and devises an optimal policy. Section 4 concludes.

\section{The market economy}

The economy is inhabited by a constant population, normalized to one, of identical individuals who derive utility from consumption, $C$, according to

$$
\int_{0}^{\infty} \frac{C^{1-\theta}-1}{1-\theta} e^{-\rho t} d t, \quad \rho>0, \quad \theta>0
$$

The endowment of time is normalized as a constant flow of one unit per period. A fraction $u_{Y}$ of time is devoted to production, a fraction $u_{H}$ to learning, and a fraction $u_{n}=1-u_{Y}-u_{H}$ to innovation. Human capital, $H$, is accumulated according to

$$
\dot{H}=\xi u_{H} H, \quad \xi>0 .
$$

The budget constraint faced by the representative individual is

$$
\dot{A}=r A+w\left(1-u_{H}\right) H-C-T,
$$

where $r$ is the return per unit of aggregate wealth $A, w$ is the wage rate per unit of employed human capital, and $T$ are lump-sum taxes (or transfers) imposed by the government. The individual maximizes her intertemporal utility (1), subject to the budget constraint (3) and the knowledge accumulation technology (2). Let $g_{\tau}$ denote $\tau$ 's growth rate, $g_{\tau}=\dot{\tau} / \tau$. The first-order conditions for an interior solution yield

$$
\begin{gathered}
g_{C}=(r-\rho) / \theta, \\
r-g_{w}=\xi,
\end{gathered}
$$

as well as the standard transversality conditions, $\lim _{t \rightarrow \infty} e^{-\rho t} \lambda A=\lim _{t \rightarrow \infty} e^{-\rho t} \mu H=0$, where $\lambda$ and $\mu$ denote the multipliers associated to constraints (3) and (2), respectively.

The market for final goods is perfectly competitive and the price for final goods is normalized to one. Final output, $Y$, is produced with a Cobb-Douglas technology

$$
Y=K^{\beta} D^{\eta}\left(u_{Y} H\right)^{1-\beta-\eta}, \quad \beta>0, \quad \eta>0, \quad \beta+\eta<1,
$$


where $K$ is the stock of physical capital and $D$ is an index of intermediate goods, $D=\left(\int_{0}^{n} x_{i}^{\alpha} d i\right)^{1 / \alpha}$, $0<\alpha<1$, where $x_{i}$ is the amount used for each one of the $n$ intermediate goods. Profit maximization delivers the factor demands

$$
\begin{aligned}
r & =\beta Y / K, \\
w & =(1-\beta-\eta) Y /\left(u_{Y} H\right), \\
p_{i} & =\eta Y x_{i}^{\alpha-1} / D^{\alpha},
\end{aligned}
$$

where $p_{i}$ represents the price of intermediate $i$.

Each firm in the intermediate goods sector owns an infinitely-lived patent for selling its variety $x_{i}$, which costs a unit of $Y$ to be produced. The government subsidizes production so that for each unit sold of the intermediate good producers receive a unit price $p_{i}$ and a subsidy $s_{x} p_{i}$. Producers act under monopolistic competition and maximize operating profits, $\pi_{i}=\left[\left(1+s_{x}\right) p_{i}-1\right] x_{i}$. Profit maximization in this sector implies that each firm charges a price of $p_{i}=1 /\left[\left(1+s_{x}\right) \alpha\right]$. Since both technology and demand are the same for all intermediates, the equilibrium is symmetric: $x_{i}=x$, $p_{i}=p$. Hence, the quantity of intermediates employed is $x n=\left(1+s_{x}\right) \alpha \eta Y$, firms profits are

$$
\pi=\left(1+s_{x}\right)(1-\alpha) \eta Y / n
$$

and $D=x n^{1 / \alpha}=n^{(1-\alpha) / \alpha}\left(1+s_{x}\right) \alpha \eta Y$. Substituting this expression into (6) yields

$$
Y^{1-\eta}=\left[\left(1+s_{x}\right) \alpha \eta\right]^{\eta} K^{\beta} n^{(1-\alpha) \eta / \alpha}\left(u_{Y} H\right)^{1-\beta-\eta} .
$$

There is free entry into the $R \& D$ sector. Invention of new intermediates is determined according to

$$
\dot{n}=\bar{\delta} u_{n} H=\delta\left(\overline{u_{n} H}\right)^{\gamma-1} n^{\phi} u_{n} H, \quad \delta>0, \quad 0<\gamma<1, \quad \phi<1,
$$

where $\overline{u_{n} H}$ represents average human capital devoted to innovation. Here, $\bar{\delta}$ is taken as given by the representative firm, so that it perceives a constant returns to scale $R \& D$ technology. This specification incorporates a duplication externality of research effort, as well as spillovers in R\&D. Three cases are possible: $\phi<0$ corresponds to the so-called "fishing-out effect" (Jones, 2005); $\phi \in(0,1)$ formalizes the "standing-on-shoulders effect", and $\phi=0$, instead, represents the situation in which the arrival rate of new ideas is independent of the available stock of inventions.

The government subsidizes a fraction $s_{R}$ of the costs of R\&D so, if $v$ is the value of an innovation, free-entry in $R \& D$ requires that

$$
\left(1-s_{R}\right) w=\delta\left(\overline{u_{n} H}\right)^{\gamma-1} n^{\phi} v
$$

where $v$ is the value of an innovation.

An innovation is worth the present value of the stream of monopoly profits, $v(t)=$ $\int_{t}^{\infty} e^{-\int_{t}^{\tau} r(s) d s} \pi(\tau) d \tau$. Differentiating this expression with respect to time yields the no-arbitrage equation

$$
g_{v}=r-\pi / v
$$

together with $\lim _{t \rightarrow \infty} v(t) e^{-\int_{s}^{t} r(\tau) d \tau}=0$.

The government may subsidize (or tax) intermediate-goods production and R\&D costs, financed by lump-sum taxation to individuals, so that its budget constraint is

$$
T=s_{x} p n x+s_{R} w u_{n} H .
$$


Henceforth we shall take into account that $\overline{u_{n} H}=u_{n} H$ in equilibrium. Physical capital and claims to innovative firms are the assets in the economy. Aggregate wealth is then $A=K+n v$. Differentiating this expression, and using (3), (7), (8), (10), (13), (14) and (15), together with $x n=\left(1+s_{x}\right) \alpha \eta Y$, we can get the economy's resource constraint

$$
g_{K}=\left[1-\left(1+s_{x}\right) \alpha \eta\right] Y / K-C / K .
$$

Some equations will be needed for solving the model. Log-differentiating (7), (8), and (11), and eliminating $g_{Y}$, we get

$$
\begin{gathered}
g_{r}=-\frac{1-\beta-\eta}{\beta} g_{w}+\frac{(1-\alpha) \eta}{\alpha \beta} g_{n}+\frac{\eta}{\beta}\left(\frac{\dot{s}_{x}}{1+s_{x}}\right), \\
g_{u_{Y}}=-\frac{1-\eta}{\beta} g_{w}+\frac{(1-\alpha) \eta}{\alpha \beta} g_{n}+g_{K}-g_{H}+\frac{\eta}{\beta}\left(\frac{\dot{s}_{x}}{1+s_{x}}\right) .
\end{gathered}
$$

Log-differentiating (13), and substituting $g_{v}$ from (14), $\pi$ from (10), $w$ from (8), and $v$ from (13), we get

$$
g_{w}=r+(\gamma-1)\left(g_{u_{n}}+g_{H}\right)-\frac{(1-\alpha)\left(1+s_{x}\right) \eta u_{Y}}{\left(1-s_{R}\right)(1-\beta-\eta) u_{n}} g_{n}+\phi g_{n}+\frac{\dot{s}_{R}}{1-s_{R}} .
$$

The system that drives the dynamics of the economy in terms of $K, H, n, C, u_{Y}$ and $u_{n}$ can be then obtained as

$$
\begin{aligned}
& g_{K}=\dot{K} / K=\left[1-\left(1+s_{x}\right) \alpha \eta\right]\left[\left(1+s_{x}\right) \alpha \eta\right]^{\frac{\eta}{1-\eta}} u_{Y}^{\frac{1-\beta-\eta}{1-\eta}}\left(\frac{H}{K}\right)^{\frac{1-\beta-\eta}{1-\eta}} n^{\frac{(1-\alpha) \eta}{\alpha(1-\eta)}}-\frac{C}{K} \\
& g_{H}=\dot{H} / H=\xi u_{H}=\xi\left(1-u_{Y}-u_{n}\right) \\
& g_{n}=\dot{n} / n=\delta u_{n}^{\gamma} H^{\gamma} n^{\phi-1} \\
& g_{C}=\dot{C} / C=\frac{1}{\theta}(r-\rho)=\frac{1}{\theta}\left[\beta\left[\left(1+s_{x}\right) \alpha \eta\right]^{\frac{\eta}{1-\eta}} u_{Y}^{\frac{1-\beta-\eta}{1-\eta}}\left(\frac{H}{K}\right)^{\frac{1-\beta-\eta}{1-\eta}} n^{\frac{(1-\alpha) \eta}{\alpha(1-\eta)}}-\rho\right] \\
& g_{u_{Y}=} \dot{u}_{Y} / u_{Y}=\left[1-\left(1+s_{x}\right) \alpha\right] \eta\left[\left(1+s_{x}\right) \alpha \eta\right]^{\frac{\eta}{1-\eta}} u_{Y}^{\frac{1-\beta-\eta}{1-\eta}}\left(\frac{H}{K}\right)^{\frac{1-\beta-\eta}{1-\eta}} n^{\frac{(1-\alpha) \eta}{\alpha(1-\eta)}} \\
& \quad+\frac{(1-\alpha) \eta}{\alpha \beta} \delta u_{n}^{\gamma} H^{\gamma} n^{\phi-1}-\frac{C}{K}-\xi\left(1-u_{Y}-u_{n}\right)+\frac{(1-\eta)}{\beta} \xi+\frac{\eta}{\beta}\left(\frac{\dot{s}_{x}}{1+s_{x}}\right) \\
& \left.g_{u_{n}=}\right) \\
& \quad \dot{u}_{n} / u_{n}=\frac{1}{1-\gamma}\left\{\xi+\left[\phi-\frac{(1-\alpha) \eta\left(1+s_{x}\right) u_{Y}}{(1-\beta-\eta)\left(1-s_{R}\right) u_{n}}\right] \delta u_{n}^{\gamma} H^{\gamma} n^{\phi-1}+\frac{\dot{s}_{R}}{1-s_{R}}\right\}-\xi\left(1-u_{Y}-u_{n}\right) .
\end{aligned}
$$

Eqs. (20), (21) and (22) come from (16), (2) and (12), respectively. Eq. (23) can be obtained from (4). From (18) and (5) we obtain (24), after substituting $g_{K}, g_{H}$ and $g_{n}$ for (20), (21) and (22), respectively. Eq. (25) is obtained by solving out in (19), using (5) and (22) to substitute for $g_{w}$ and $g_{n}$, respectively. 
Equations (20)-(25) plus the transversality conditions and the initial conditions $-K(0)$, $H(0)$ and $n(0)$ - determine the transitional dynamics, by jointly determining the variables $\left(K(t), H(t), n(t), C(t), u_{Y}(t), u_{n}(t)\right) .^{3}$

Both policies $s_{x}$ and $s_{R}$ affect the dynamics of the economy in levels and growth rates. For example, the level of $s_{x}$ would increase the consumption growth rate due to its effect in decreasing the price of intermediates - used as a factor of production for the consumption final good. Also, the growth rate of $s_{x}$ is positively related to the growth rate of the allocation of human capital to the final good production $\left(g_{u_{Y}}\right)$ which is related to the positive effect this subsidy has on final good production. The effect of $s_{x}$ on the growth rate of physical capital is non-linear as it tends to increase production through its effect of intermediate goods but also tends to increase consumption due to its effect on price. Moreover, it acts to decrease the growth rate of $u_{n}$ because it increases the opportunity cost of investing in $\mathrm{R} \& \mathrm{D}$. The effect of the $\mathrm{R} \& \mathrm{D}$ subsidy growth rate on the growth rate of human capital allocated to the innovative sector $\left(g_{u_{n}}\right)$ is positive, which is explained by the increasing R\&D sector demand for human capital when this subsidy is introduced. There are additional effects from $s_{x}$ and $s_{R}$ on $g_{u_{Y}}$ and $g_{u_{n}}$ which sign cannot be analytically analyzed as there are terms of opposite signs.

\section{The socially planned economy}

This section analyses the Pareto-efficient solution for this model. The social planner chooses all quantities directly, taking all the relevant information into account. Since the intermediategoods sector is symmetric, the planner will employ the quantity $x_{i}=x$ of each good. Hence, the production function can be rewritten as $Y=K^{\beta} n^{\eta / \alpha} x^{\eta}\left(u_{Y} H\right)^{1-\beta-\eta}$, and the resources constraint is

$$
\dot{K}=Y-C-\int_{0}^{n} x_{i} d i=K^{\beta} n^{\eta / \alpha} x^{\eta}\left(u_{Y} H\right)^{1-\beta-\eta}-C-n x .
$$

\subsection{Optimal growth path}

The planner seeks to maximize (1) subject to (26), (2) and (12). Let $\mathcal{H}$ be the current value Hamiltonian, and let $\lambda, \mu$ and $\varphi$ be the multipliers for the constraints (26), (2) and (12), respectively:

$$
\mathcal{H}=\frac{C^{1-\theta}-1}{1-\theta}+\lambda(Y-n x-C)+\mu \xi\left(1-u_{Y}-u_{n}\right) H+\varphi \delta\left(u_{n} H\right)^{\gamma} n^{\phi} .
$$

Here, the control variables are $C, x, u_{Y}, u_{H}$, and $K, H$ and $n$ are the state variables. The first-order conditions for an interior solution are

$$
\begin{gathered}
C^{-\theta}=\lambda, \\
\eta Y / x=n, \\
\lambda(1-\beta-\eta) Y / u_{Y}=\mu \xi H, \\
\mu \xi H=\varphi \gamma n g_{n} / u_{n}, \\
\dot{\lambda}=(\rho-\beta Y / K) \lambda, \\
\dot{\mu}=\rho \mu-\lambda(1-\beta-\eta) Y / H-\mu \xi\left(1-u_{Y}-u_{n}\right)-\varphi \gamma n g_{n} / H,
\end{gathered}
$$

\footnotetext{
${ }^{3}$ The dynamics of the decentralized economy could be easily expressed in terms of variables that are constant in the balanced growth path in a similar manner as it will be done in Section 3 (see also Gómez, 2011a). However, this will not be necessary for our purposes.
} 


$$
\dot{\varphi}=\rho \varphi-\lambda[\eta Y /(\alpha n)-x]-\varphi \phi g_{n},
$$

plus the usual transversality conditions

$$
\lim _{t \rightarrow \infty} e^{-\rho t} \lambda K=\lim _{t \rightarrow \infty} e^{-\rho t} \mu H=\lim _{t \rightarrow \infty} e^{-\rho t} \varphi n=0 .
$$

There are two main qualitative differences between the market equilibrium and the first-best optimum. First, the planner internalizes the inefficiency due to the presence of monopolistic competition in intermediate-goods production. Therefore, he chooses to devote to intermediate-goods production a fraction of output equal to the elasticity of intermediates in the production of the final good, $x n / Y=\eta$. Second, the planner internalizes the spillovers in R\&D and the duplication externalities. This is taken into account when choosing the optimal fraction of time devoted to innovation in (31) and the optimal shadow value of an innovation in (34).

From (31), (30), (33) and (34) we get

$$
\begin{gathered}
g_{\mu}=\rho-\xi \\
g_{\varphi}=\rho-\left[\frac{\eta(1-\alpha) \gamma u_{Y}}{\alpha(1-\beta-\eta) u_{n}}+\phi\right] g_{n} .
\end{gathered}
$$

Substituting $x$ from (29) into the production function, we obtain

$$
Y^{1-\eta}=\eta^{\eta} K^{\beta} n^{(1-\alpha) \eta / \alpha}\left(u_{Y} H\right)^{1-\beta-\eta} .
$$

From (26) and (29), the resources constraint can be expressed as

$$
g_{K}=(1-\eta) Y / K-C / K .
$$

Log-differentiating (28), (31), (30), (38) and (12) we get

$$
\begin{gathered}
g_{\lambda}=-\theta g_{C} \\
g_{\varphi}=g_{\mu}-g_{g_{n}}-g_{n}+g_{u_{n}}+g_{H}, \\
g_{\mu}=g_{\lambda}+g_{Y}-g_{u_{Y}}-g_{H} \\
(1-\eta) g_{Y}=\beta g_{K}+[(1-\alpha) \eta / \alpha] g_{n}+(1-\beta-\eta)\left(g_{u_{Y}}+g_{H}\right), \\
g_{g_{n}}=\gamma\left(g_{u_{n}}+g_{H}\right)-(1-\phi) g_{n} .
\end{gathered}
$$

The system that drives the dynamics of the economy in terms of $K, H, n, C, u_{Y}$ and $u_{n}$ can then be obtained as

$$
\begin{aligned}
g_{K} & =\dot{K} / K=(1-\eta) \frac{Y}{K}-\frac{C}{K}=(1-\eta) \eta^{\frac{\eta}{1-\eta}} u_{Y}^{\frac{1-\beta-\eta}{1-\eta}}\left(\frac{H}{K}\right)^{\frac{1-\beta-\eta}{1-\eta}} n^{\frac{(1-\alpha) \eta}{\alpha(1-\eta)}}-\frac{C}{K} \\
g_{H} & =\dot{H} / H=\xi u_{H}=\xi\left(1-u_{Y}-u_{n}\right) \\
g_{n} & =\dot{n} / n=\delta u_{n}^{\gamma} H^{\gamma} n^{\phi-1} \\
g_{C} & =\dot{C} / C=\frac{1}{\theta}\left[\beta \frac{Y}{K}-\rho\right]=\frac{1}{\theta}\left[\beta \eta^{\frac{\eta}{1-\eta}} u_{Y}^{\frac{1-\beta-\eta}{1-\eta}}\left(\frac{H}{K}\right)^{\frac{1-\beta-\eta}{1-\eta}} n^{\frac{(1-\alpha) \eta}{\alpha(1-\eta)}}-\rho\right] \\
g_{u_{Y}} & =\dot{u}_{Y} / u_{Y}=\frac{(1-\alpha) \eta}{\alpha \beta} \delta u_{n}^{\gamma} H^{\gamma} n^{\phi-1}-\frac{C}{K}-\xi\left(1-u_{Y}-u_{n}\right)+\frac{1-\eta}{\beta} \xi
\end{aligned}
$$




$$
g_{u_{n}}=\dot{u}_{n} / u_{n}=-\frac{(1-\alpha) \eta \gamma \delta u_{Y} H^{\gamma} n^{\phi-1} u_{n}^{\gamma-1}}{\alpha(1-\beta-\eta)(1-\gamma)}-\xi\left(1-u_{Y}-u_{n}\right)+\frac{\xi}{1-\gamma} .
$$

Eqs. (45), (46) and (47) come from (39), (2) and (12), respectively. Eq. (48) can be obtained from (32) and (40). Solving for $g_{Y}-g_{u_{Y}}-g_{H}$ in (42) and (43), equating the resulting expressions, and solving out for $g_{u_{Y}}$ we obtain (49), after eliminating $g_{\lambda}$ and $g_{\mu}$ by means of (32) and (36), respectively, and substituting $g_{K}$ and $g_{H}$ for (45) and (46). Eq. (50) is obtained by substituting $g_{g_{n}}$ for (44) in (41), solving out for $g_{u_{n}}$, and using (36), (37), (46) and (47) so substitute for $g_{\mu}, g_{\varphi}$, $g_{H}$ and $g_{n}$, respectively.

Our next step, as it is usual in the endogenous growth literature, will be to express the dynamics of the economy in terms of variables that are constant in the balanced growth path (BGP). It is evident that $g_{u_{Y}}=g_{u_{n}}=0$, i.e., that $u_{Y}$ and $u_{n}$ are constant in the BGP because, otherwise, they would violate the bound constraints $0 \leq u_{Y}, u_{n} \leq 1$. The expression for $g_{n}$ in Eq. (47) entails that the 'knowledge-ideas' ratio $\psi \equiv H^{\gamma} n^{\phi-1}$ must be constant in the BGP, where $\psi$ is a predetermined variable because $H$ and $n$ are so. Eq. (48) implies that $q=(H / K)^{\frac{1-\beta-\eta}{1-\eta}} n^{\frac{(1-\alpha) \eta}{\alpha(1-\eta)}}$ is constant in the BGP, where the variable $q$ is also predetermined because $K, H$ and $n$ are so. Finally, Eq. (45) entails that $\chi=C / K$ must also be constant in the BGP. Note that if $u_{Y}$ and $u_{n}$ are constant, Eq. (46) entails that the growth rate of $H$ is also constant in the BGP.

Hence, the dynamics of the economy can be described in terms of variables that are constant in the BGP by considering the predetermined variables $\psi \equiv H^{\gamma} n^{\phi-1}$ and $q=(H / K)^{\frac{1-\beta-\eta}{1-\eta}} n^{\frac{(1-\alpha) \eta}{\alpha(1-\eta)}}$, and the jump variables $\chi=C / K, u_{Y}$ and $u_{n}$. Using that

$$
\begin{aligned}
g_{q} & =\frac{1-\beta-\eta}{1-\eta}\left(g_{H}-g_{K}\right)+\frac{(1-\alpha) \eta}{\alpha(1-\eta)} g_{n}, \\
g_{\psi} & =\gamma g_{H}-(1-\phi) g_{n}, \\
g_{\chi} & =g_{C}-g_{K},
\end{aligned}
$$

and taking into account (45)-(48), we can eventually get the system ${ }^{4}$

$$
\begin{aligned}
g_{q} & =\frac{1-\beta-\eta}{1-\eta}\left[\xi\left(1-u_{Y}-u_{n}\right)-(1-\eta) \eta^{\frac{\eta}{1-\eta}} u_{Y}^{\frac{1-\beta-\eta}{1-\eta}} q+\chi\right]+\frac{(1-\alpha) \eta}{\alpha(1-\eta)} \delta u_{n}^{\gamma} \psi, \\
g_{\psi} & =\gamma \xi\left(1-u_{Y}-u_{n}\right)-(1-\phi) \delta u_{n}^{\gamma} \psi, \\
g_{\chi} & =\frac{1}{\theta}\left[\beta \eta^{\frac{\eta}{1-\eta}} u_{Y}^{\frac{1-\beta-\eta}{1-\eta}} q-\rho\right]-(1-\eta) \eta^{\frac{\eta}{1-\eta}} u_{Y}^{\frac{1-\beta-\eta}{1-\eta}} q+\chi, \\
g_{u_{Y}} & =\frac{(1-\alpha) \eta}{\alpha \beta} \delta u_{n}^{\gamma} \psi-\chi-\xi\left(1-u_{Y}-u_{n}\right)+\frac{1-\eta}{\beta} \xi \\
g_{u_{n}} & =-\frac{(1-\alpha) \eta \gamma \delta u_{Y} \psi u_{n}^{\gamma-1}}{\alpha(1-\beta-\eta)(1-\gamma)}-\xi\left(1-u_{Y}-u_{n}\right)+\frac{\xi}{1-\gamma} .
\end{aligned}
$$

\footnotetext{
${ }^{4}$ The change of variables made satisfy conditions (7)-(9) in Martínez-García (2003), -i.e., the new variables explain their own dynamics, as it happens in system (54)-(58) (or in system (59)-(63)), when this system is evaluated at the BGP the result is a steady state, and they explain the growth rates of the state and costate variables-, as well as the conditions in her Lemmas 1 and 2. Hence, analyzing the existence of paths converging to the BGP is equivalent to studying the stability of the steady state in (54)-(58) (or (59)-(63)). The reduction of dimension is a standard practice in the endogenous growth literature (e.g., Benhabib and Perli, 1994; Barro and Sala-i-Martin, 1995; Benhabib et al., 2000; Mino, 2001; Arnold, 2006).
} 
Here, we have used that $(38)$ entails that $Y / K=\eta^{\frac{\eta}{1-\eta}} u_{Y}^{\frac{1-\beta-\eta}{1-\eta}}(H / K)^{\frac{1-\beta-\eta}{1-\eta}} n^{\frac{(1-\alpha) \eta}{\alpha(1-\eta)}}=\eta^{\frac{\eta}{1-\eta}} u_{Y}^{\frac{1-\beta-\eta}{1-\eta}} q$.

However, the complexity of system (54)-(58), and of its jacobian matrix, would make difficult (if possible) to obtain analytical results on the (local) stability. Hence, we shall make use of a dynamic system in terms of the variables $r=\beta Y / K=\beta \eta^{\frac{\eta}{1-\eta}} u_{Y}^{\frac{1-\beta-\eta}{1-\eta}} q, \chi=C / K, g_{n}=\delta u_{n}^{\gamma} H^{\gamma} n^{\phi-1}=\delta u_{n}^{\gamma} \psi$, $z=\delta^{1 / \gamma} \psi^{1 / \gamma} u_{Y}$, and $u_{Y}$. So, using (54)-(58), we eventually find that the dynamics of the economy is driven by the system

$$
\begin{aligned}
g_{r}= & \frac{1-\beta-\eta}{1-\eta} g_{u_{Y}}+g_{q}=\frac{1-\beta-\eta}{\beta}(\xi-r)+\frac{(1-\alpha) \eta}{\alpha \beta} g_{n}, \\
g_{\chi}= & \left(\frac{1}{\theta}-\frac{1-\eta}{\beta}\right) r+\chi-\frac{\rho}{\theta} \\
g_{g_{n}}= & \gamma g_{n}+g_{\psi}=-\frac{(1-\alpha) \eta \gamma^{2} g_{n}^{1-1 / \gamma} z}{\alpha(1-\beta-\eta)(1-\gamma)}-(1-\phi) g_{n}+\frac{\gamma \xi}{1-\gamma} \\
g_{z}= & (1 / \gamma) g_{\psi}+g_{u_{Y}}=\left[\frac{(1-\alpha) \eta}{\alpha \beta}-\frac{1-\phi}{\gamma}\right] g_{n}-\chi+\frac{1-\eta}{\beta} \xi \\
g_{u_{Y}}= & \frac{(1-\alpha) \eta}{\alpha \beta} g_{n}-\chi-\xi\left(1-u_{Y}-\frac{u_{Y} g_{n}^{1 / \gamma}}{z}\right)+\frac{1-\eta}{\beta} \xi .
\end{aligned}
$$

\subsection{Steady state}

Now, we focus on a balanced growth path (or steady state) in which all variables grow at constant rates, and the shares of labor in its different uses are constant.

Proposition 1. Let $\xi>\rho$. The centralized economy has a unique positive steady state with positive long-run growth,

$$
\begin{gathered}
\bar{r}=\frac{(1+M) \theta \xi-\rho}{(1+M) \theta-1}, \\
\bar{\chi}=\left(\frac{1-\eta}{\beta}-\frac{1}{\theta}\right) \bar{r}+\frac{\rho}{\theta}, \\
\bar{g}_{n}=\frac{\gamma M(\xi-\rho)}{(1-\phi)[(1+M) \theta-1]}, \\
\bar{g}_{H}=(1-\phi) \bar{g}_{n} / \gamma, \\
\bar{u}_{n}=\frac{(1-\alpha) \eta \gamma \bar{g}_{n}\left(1-\bar{g}_{H} / \xi\right)}{\alpha(1-\beta-\eta)\left[\xi-\bar{g}_{H}+(1-\phi) \bar{g}_{n}\right]+(1-\alpha) \eta \gamma \bar{g}_{n}}, \\
\bar{u}_{Y}=1-\bar{u}_{n}-\bar{g}_{H} / \xi \\
\bar{\psi}=\bar{g}_{n} /\left(\delta \bar{u}_{n}^{\gamma}\right), \\
\bar{z}=\delta^{1 / \gamma} \bar{\psi}^{1 / \gamma} \bar{u}_{Y}, \\
\bar{q}=\eta^{-\frac{\eta}{1-\eta}} \bar{u}_{Y}^{-\frac{1-\beta-\eta}{1-\eta}} \bar{r} / \beta, \\
\bar{g}_{Y}=\bar{g}_{C}=\bar{g}_{K}=(1+1 / M) \bar{g}_{H},
\end{gathered}
$$

where $M=\alpha(1-\beta-\eta)(1-\phi) /[(1-\alpha) \eta \gamma]$, if and only if

$$
\theta>\theta_{\min }=\frac{1+M(1-\rho / \xi)}{1+M}
$$


Proof. Evaluating (2), (60) and (44) at the steady state we obtain (69), (65) and (67), respectively. From (43), using (67), we get (73). From (28 and (32) we get $\bar{g}_{C}=(\bar{r}-\rho) / \theta$. Now using $(67)$ and (73) to express $\bar{g}_{C}$ as a function of $\bar{g}_{n}$ and solving the resulting equation and (59) for $\bar{r}$ and $\bar{g}_{n}$, yields (64) and (66). Eq. (68) results from equating (37) and (41) and solving out for $u_{n}$, using (36) and (69), and (70) is obtained from (12). Finally, (71) and (72) results from their definitions.

For the interior steady state to be feasible, we must have $0<\bar{u}_{Y}, 0<\bar{u}_{n}, \bar{u}_{Y}+\bar{u}_{n}<1, \bar{r}>0$, $\bar{\chi}>0$ and $\bar{\psi}>0$. Eqs. (67) and (66) entail that condition $0<\bar{u}_{Y}+\bar{u}_{n}=\left(\xi-\bar{g}_{H}\right) / \xi<1$ is satisfied if and only if (74) holds. Since (74) entails that $\theta>1 /(1+M)$, Eqs. (64) and (66) entail that $\bar{r}>0$ and $\bar{g}_{n}>0$ if $\xi>\rho$. Furthermore, $\bar{u}_{Y}$ and $\bar{u}_{n}$ are positive because $\xi-\bar{g}_{H}>0$. Hence, Eq. (70) entails that $\bar{\psi}>0$. Finally, the ratio of consumption to capital can be expressed as

$$
\bar{\chi}=\frac{(1-\eta)[(1+M) \theta \xi-\rho]-\beta(1+M)(\xi-\rho)}{\beta[(1+M) \theta-1]} .
$$

The denominator is positive and, as condition (74) can be equivalently rewritten as $(1+M) \theta \xi>$ $\xi+M(\xi-\rho)$, the numerator is also positive because it is greater than $(1-\beta-\eta)(1+M)(\xi-\rho)>0$, and so $\bar{\chi}>0$. Hence, the steady state is feasible.

The transversality condition associated to $K$ is equivalent to $-\rho+\bar{g}_{\lambda}+\bar{g}_{K}=-\xi+\bar{g}_{H}<0$, using (42) and (36), and taking into account that $\bar{g}_{K}=\bar{g}_{Y}$. Using (36), the transversality condition associated to $H$ is equivalent to $-\rho+\bar{g}_{\mu}+\bar{g}_{H}=-\xi+\bar{g}_{H}<0$. Using (41), the transversality condition associated to $n$ is equivalent to $-\rho+g_{\varphi}+g_{n}=-\xi+\bar{g}_{H}<0$. This completes the proof.

It should be noted that the condition $\phi<1$ is required for an interior BGP with positive longrun growth to exist, as it results clear from (67). Furthermore, this is also the usual condition in the class of models in the line of Jones (1995) and Arnold (1998).

The following proposition analyzes the (local) stability of the steady state. Although the stability analysis has been mostly neglected in the related literature, this analysis is important because, in absence of stability, convergence to the first-best solution cannot be guaranteed which would make this outcome meaningless. However, stability cannot be taken for granted in this model and, actually, the first-best stationary solution is unstable in the model with no duplication externalities. $^{5}$

Proposition 2. In the conditions of Proposition 1, a) the steady state of the centralized economy is either saddle-path stable or unstable, and b) a sufficient condition to rule out the instability outcome is

$$
\alpha \beta(1-\phi) \geq(1-\alpha) \eta \gamma
$$

Proof. The stocks of physical and human capital, and the number of intermediates move sluggishly, so that $K(0), H(0)$ and $n(0)$ are given by their historical values. Therefore, $q$ and $\psi$ are predetermined variables. with $q(0)=(H(0) / K(0))^{(1-\beta-\eta) /(1-\eta)} n(0)^{[(1-\alpha) \eta] /[\alpha(1-\eta)]}$ and $\psi(0)=$ $H(0)^{\gamma} n(0)^{\phi-1}$. So, the dynamic system (54)-(58) in the variables $\psi, q, \chi, u_{Y}$ and $u_{n}$ features two predetermined variables $\left(q\right.$ and $\psi$ ) and three jump variables $\left(\chi, u_{Y}\right.$ and $\left.u_{n}\right)$, so saddle-path stability would require two stable roots.

\footnotetext{
${ }^{5}$ The proof for this specific case is in Appendix A.
} 
When we consider the dynamic system (59)-(63), it could seem that the variables $r, \chi, g_{n}, z$ and $u_{Y}$ are all jumpable. However, they cannot really jump independently and freely because they must satisfy the following two conditions

$$
\begin{aligned}
& r(0)=\beta \eta^{\frac{\eta}{1-\eta}} u_{Y}(0)^{\frac{1-\beta-\eta}{1-\eta}} q(0), \\
& z(0)=\delta^{1 / \gamma} \psi(0)^{1 / \gamma} u_{Y}(0),
\end{aligned}
$$

Hence, there are only three degrees of freedom: we can think as if only three variables can jump freely - say $\chi, g_{n}$ and $u_{Y}$-, whereas the other two variables - say $r$ and $z$-, are then determined by the former constraints (76) and (77). So, saddle-path stability (local determinacy) requires two stable roots as it happened with system (54)-(58)

Linearization of (59)-(63) around the steady state yields

$$
\left(\begin{array}{c}
\dot{r} \\
\dot{\chi} \\
\dot{g}_{n} \\
\dot{z} \\
\dot{u}_{Y}
\end{array}\right)=\left(\begin{array}{ccccc}
J_{11} & 0 & J_{13} & 0 & 0 \\
J_{21} & J_{22} & 0 & 0 & 0 \\
0 & 0 & J_{33} & J_{34} & 0 \\
0 & J_{42} & J_{43} & 0 & 0 \\
\bullet & \bullet & \bullet & \bullet & J_{55}
\end{array}\right)\left(\begin{array}{c}
r-\bar{r} \\
\chi-\bar{\chi} \\
g_{n}-\bar{g}_{n} \\
z-\bar{z} \\
u_{Y}-\bar{u}_{Y}
\end{array}\right)=J \cdot\left(\begin{array}{c}
r-\bar{r} \\
\chi-\bar{\chi} \\
g_{n}-\bar{g}_{n} \\
z-\bar{z} \\
u_{Y}-\bar{u}_{Y}
\end{array}\right),
$$

where dots replace those elements that are irrelevant for the analysis, and

$$
\begin{array}{ll}
J_{11}=-\frac{1-\beta-\eta}{\beta} \bar{r}<0, & J_{13}=\frac{(1-\alpha) \eta}{\alpha \beta} \bar{r}>0, \\
J_{21}=\left(\frac{1}{\theta}-\frac{1-\eta}{\beta}\right) \bar{\chi}, & J_{22}=\bar{\chi}>0, \\
J_{33}=\xi-(1-\phi) \bar{g}_{n} / \gamma, & J_{34}=-\frac{(1-\alpha) \eta \gamma^{2}}{(1-\gamma) \alpha(1-\beta-\eta)} \bar{g}_{n}^{2-1 / \gamma}<0, \\
J_{42}=-\bar{z}<0, & J_{43}=\left[\frac{(1-\alpha) \eta}{\alpha \beta}-\frac{1-\phi}{\gamma}\right] \bar{z}, \\
J_{55}=\xi\left(1+\bar{g}_{n}^{1 / \gamma} / \bar{z}\right) \bar{u}_{Y}>0 . &
\end{array}
$$

The eigenvalues of $J$ are the four eigenvalues of its upper left $4 \times 4$ submatrix (say, $\bar{J}$ ) and its last diagonal element, $J_{55}>0$. Therefore, the number of stable roots of $J$ is equal to that of $\bar{J}$. Using the Routh-Hurwitz theorem, the number of roots of the characteristic equation of $\bar{J}$, $p(x)=x^{4}-\Delta_{3} x^{3}+\Delta_{2} x^{2}-\Delta_{1} x+\Delta_{0}=0$, with negative real parts is equal to the number of variations of sign in the scheme:

$$
\begin{array}{lllll}
1 & \Delta_{3} & \Psi & \Pi & \Delta_{0}
\end{array}
$$

where

$$
\begin{aligned}
\Delta_{0} & =\operatorname{det}(\bar{J})=\frac{(1-\alpha) \eta \gamma^{2}(\xi-\rho)}{\alpha \beta \theta(1-\gamma)} \bar{z} \bar{r} \bar{\chi} \bar{g}_{n}^{1-1 / \gamma}>0, \\
\Delta_{1} & =J_{11} J_{22} J_{33}-\left(J_{11}+J_{22}\right) J_{34} J_{43}, \\
\Delta_{2} & =\left(J_{11}+J_{22}\right) J_{33}+J_{11} J_{22}-J_{34} J_{43}, \\
\Delta_{3} & =\operatorname{tr}(\bar{J})=J_{11}+J_{22}+J_{33}, \\
\Psi & =\Delta_{2}-\Delta_{1} / \operatorname{tr}(\bar{J}),
\end{aligned}
$$




$$
\Pi=\Delta_{1}-\left[\Delta_{3} \operatorname{det}(\bar{J}) / \Psi\right],
$$

It is easy to see that $\xi-\bar{g}_{H}>0$ if condition (74) holds, so that

$$
\begin{aligned}
J_{11}+J_{22} & =\bar{r}-\bar{g}_{K}=\xi-\bar{g}_{H}>0, \\
J_{33} & =\xi-(1-\phi) \bar{g}_{n} / \gamma=\xi-\bar{g}_{H}>0,
\end{aligned}
$$

and, therefore, $\Delta_{3}=\operatorname{tr}(\bar{J})>0$. Given the positivity of the determinant and the trace, there can be at most two variations of sign in the scheme (79). Hence, the matrix $\bar{J}$ may have 0 or 2 roots with negative real parts. This proves part a).

To prove part b), we first show that a sufficient condition to rule out the case of none stable roots is that $\Delta_{1}<0$. If $\Psi<0$, there are two variations in sign in (79) -irrespective of the sign of $\Pi$. If $\Psi>0$ then $\Pi<0$ and, therefore, there are two variations in sign in (79). If $\Psi=0$, we substitute it by $\Psi=\iota>0$, and so, $\Pi=\Delta_{1}-\operatorname{tr}(\bar{J}) \operatorname{det}(\bar{J}) / \iota$. Taking the limit as $\iota \rightarrow 0$, we have that $\Pi \rightarrow-\infty$ and, therefore, there are two variations in sign in (79). Given that $J_{11}+J_{22}>0$ and $J_{33}>0$, the expression for $\Delta_{1}$ indicates that a sufficient condition for $\Delta_{1}<0$ is that $J_{43} \leq 0$; i.e., that condition (75) holds.

As in the case of the market economy (see Gómez, 2011a), instability cannot be ruled out in the socially planned economy. For example, the parameterization $\beta=0.2, \eta=0.6, \alpha=0.4, \xi=0.05$, $\rho=0.02, \theta=1.1, \delta=0.1, \phi=0.8, \gamma=0.95$ yields a feasible steady state. The eigenvalues of the linearized system are $0.0204 \pm 0.2895 i, 0.0204 \pm 0.1464 i$, and 0.0407 , and so, the optimal steady state is instable.

\subsection{Optimal policy}

This section devises a fiscal policy capable of making the market equilibrium described by (20)(25) to replicate the first-best optimum described by (45)-(50). The following proposition states an optimal policy for this economy.

Proposition 3. In the conditions of Proposition 2, the first-best solution can be decentralized by means of a subsidy to intermediate-goods production at a constant rate $s_{x}=(1-\alpha) / \alpha$, combined with a time-varying subsidy (or tax) to REDD that evolves according to

$$
\dot{s}_{R}=\left[\frac{(1-\alpha) \eta}{\alpha(1-\beta-\eta)}\left[1-\gamma\left(1-s_{R}\right)\right] \frac{u_{Y}}{u_{n}}-\left(1-s_{R}\right) \phi\right] g_{n},
$$

and converges in the long-run to

$$
\bar{s}_{R}=1-\frac{(1-\alpha) \eta \bar{u}_{Y} / \bar{u}_{n}}{\alpha(1-\beta-\eta) \phi+\gamma(1-\alpha) \eta \bar{u}_{Y} / \bar{u}_{n}},
$$

which are financed by means of lump-sum taxation.

Proof. Note first that the laws of motion of $H$ and $n$ in the market economy coincide with their counterparts in the socially planned economy. Now, comparing the laws of motion of $C$ in the market and the socially-planned economies, given by (23) and (48), we get that

$$
s_{x}=(1-\alpha) / \alpha .
$$

Hence, $s_{x}$ is constant and, therefore, $\dot{s}_{x}=0$. Note that introducing a subsidy to production $s_{x}$ makes the total quantities of intermediates to be $n x=\left(1+s_{x}\right) \alpha \eta Y$. In order to achieve the optimal 
quantity in (29), the subsidy rate has to be set according to (80). In this way, (20) and (24) also coincide with (45) and (49). Finally, comparing (25) with (50), and using (80), we get that the optimal subsidy to R\&D must follow the law of motion

$$
\dot{s}_{R}=\left[\frac{(1-\alpha) \eta}{\alpha(1-\beta-\eta)}\left[1-\gamma\left(1-s_{R}\right)\right] \frac{u_{Y}}{u_{n}}-\left(1-s_{R}\right) \phi\right] \delta u_{n}^{\gamma} H^{\gamma} n^{\phi-1} .
$$

Using that $g_{n}=\delta u_{n}^{\gamma} H^{\gamma} n^{\phi-1}$, this can be equivalently expressed as the expression for $\dot{s}_{R}$ in the proposition.

We have already shown that the market economy replicates the first-best optimum. Therefore, it remains to show that the optimal subsidy to R\&D indeed converges to its long-run value $\bar{s}_{R}$. To this end, we first rewrite the law of motion of $s_{R}$ as

$$
\dot{s}_{R}=\frac{(1-\alpha) \eta}{\alpha(1-\beta-\eta)}\left[1-\gamma\left(1-s_{R}\right)\right] g_{n}^{1-1 / \gamma} z-\left(1-s_{R}\right) \phi g_{n}
$$

using that $u_{n}=g_{n}^{1 / \gamma} u_{Y} / z$, where the evolution of $g_{n}, u_{Y}$ and $z$ is determined by the system (59)-(63). Linearizing the system (59)-(63), together with (82), around its steady state $\left(\bar{r}, \bar{\chi}, \bar{g}_{n}, \bar{z}, \bar{u}_{Y}, \bar{s}_{R}\right)$, the jacobian matrix is

$$
\tilde{J}=\left(\begin{array}{cc}
J & \mathbf{0} \\
J_{R} & J_{66}
\end{array}\right)
$$

where $J$ is defined in (78), $J_{R}$ is a row matrix of order $1 \times 5$ whose elements are irrelevant for the analysis, $\mathbf{0}$ is a column matrix of zeros of order $5 \times 1$, and

$$
J_{66}=\frac{\partial \dot{s}_{R}}{\partial s_{R}}=\frac{\eta(1-\alpha) \bar{g}_{n} \bar{u}_{Y} / \bar{u}_{n}}{\left(1-\bar{s}_{R}\right) \alpha(1-\beta-\eta)}>0 .
$$

Therefore, the number of stable roots of the matrix $\tilde{J}$ is equal to that of the matrix $J$ and, since $s_{R}$ is a jump variable, saddle-path stability is guaranteed if there are two stable roots. Hence, the results stated in Proposition 2 remain valid.

It is interesting to note that equation for $\dot{s}_{R}(81)$ entails that subsidy to R\&D should rise more the greatest the difference between allocation of human capital to the final good and to the R\&D sector, i.e., the higher the ratio $u_{Y} / u_{n}$, the higher $\dot{s}_{R}$.

In the absence of $\mathrm{R} \& \mathrm{D}$ spillovers, $\phi=0$, the long-run optimal subsidy rate is $\bar{s}_{R}=-(1-\gamma) / \gamma<$ 0 , whereas in the absence of duplication externalities, $\gamma=1$, the optimal subsidy/tax becomes $\bar{s}_{R}=\alpha(1-\beta-\eta) \phi /\left[\alpha(1-\beta-\eta) \phi+(1-\alpha) \eta \bar{u}_{Y} / \bar{u}_{n}\right]$, which is positive or negative depending on whether there are positive or negative R\&D spillovers.

The fact that the equilibrium is saddle-point stable and the linearized stable manifold is twodimensional has the interesting consequence that the transitional dynamics and, in particular, the time path of the optimal $R \& D$ subsidy can, under certain circumstances, be non-monotonic even without complex eigenvalues of the linearised system (e.g., Brito and Dixon, 2009). In fact, for a number of realistic sets of calibrated values, we can observe that the R\&D subsidy may evolve through smooth or damped oscillations. This result is in sharp contrast with the analytical results in Arnold (2000b) and the numerical results in Grossmann et al. (2010), which find monotonic paths for the optimal R\&D subsidy.

The effect of externalities associated to $R \& D$ on the long-run value of the subsidy to $R \& D$ is stated in the following proposition. 
Proposition 4. An increase in RESD spillovers increases the long-run optimal subsidy to RED, and an increase in duplication externalities decreases the long-run optimal subsidy.

Proof. Using that $\bar{u}_{Y}=1-\bar{u}_{n}-\bar{g}_{H} / \xi$ and (68), and using (67) to express $\bar{g}_{n}$ as a function of $\bar{g}_{H}$ - which does not depend on $\gamma$ or $\phi$-, we obtain that

$$
\frac{\bar{u}_{Y}}{\bar{u}_{n}}=\frac{\alpha(1-\beta-\eta)(1-\phi)\left(\xi-\bar{g}_{H}+\gamma \bar{g}_{H}\right)}{\gamma^{2} \eta(1-\alpha) \bar{g}_{H}} .
$$

Partial derivation of $\bar{s}_{R}$ with respect to $\phi$ and $\gamma$, holding constant the ratio $\bar{u}_{Y} / \bar{u}_{n}$, entails that

$$
\begin{aligned}
& \left.\frac{\partial \bar{s}_{R}}{\partial \phi}\right|_{\bar{u}_{Y} / \bar{u}_{n}=\mathrm{constant}}=\frac{\alpha(1-\beta-\eta) \bar{u}_{n}}{\eta(1-\alpha) \bar{u}_{Y}}\left(1-\bar{s}_{R}\right)^{2}>0, \\
& \left.\frac{\partial \bar{s}_{R}}{\partial \gamma}\right|_{\bar{u}_{Y} / \bar{u}_{n}=\mathrm{constant}}=\left(1-\bar{s}_{R}\right)^{2}>0,
\end{aligned}
$$

whereas the derivative of $\bar{s}_{R}$ with respect to $\bar{u}_{Y} / \bar{u}_{n}$ is

$$
\frac{\partial \bar{s}_{R}}{\partial\left(\bar{u}_{Y} / \bar{u}_{n}\right)}=-\frac{\alpha(1-\beta-\eta) \phi \bar{u}_{n}^{2}}{\eta(1-\alpha) \bar{u}_{Y}^{2}}\left(1-\bar{s}_{R}\right)^{2} .
$$

Now, the derivatives of $\bar{u}_{Y} / \bar{u}_{n}$ with respect to $\phi$ and $\gamma$ are

$$
\begin{aligned}
\frac{\partial\left(\bar{u}_{Y} / \bar{u}_{n}\right)}{\partial \phi} & =-\frac{\bar{u}_{Y}}{(1-\phi) \bar{u}_{n}}<0 \\
\frac{\partial\left(\bar{u}_{Y} / \bar{u}_{n}\right)}{\partial \gamma} & =-\frac{\left[2\left(\xi-\bar{g}_{H}\right)+\gamma \bar{g}_{H}\right] \bar{u}_{Y}}{\gamma\left(\xi-\bar{g}_{H}+\gamma \bar{g}_{H}\right) \bar{u}_{n}}<0 .
\end{aligned}
$$

On the effect of spillovers in $R \& D$, after simplification, we have that

$$
\frac{\partial \bar{s}_{R}}{\partial \phi}=\left.\frac{\partial \bar{s}_{R}}{\partial \phi}\right|_{\bar{u}_{Y} / \bar{u}_{n}=\mathrm{constant}}+\frac{\partial \bar{s}_{R}}{\partial\left(\bar{u}_{Y} / \bar{u}_{n}\right)} \frac{\partial\left(\bar{u}_{Y} / \bar{u}_{n}\right)}{\partial \phi}=\frac{\alpha(1-\beta-\eta) \bar{u}_{n}}{\eta(1-\alpha)(1-\phi) u_{Y}}\left(1-\bar{s}_{R}\right)^{2}>0
$$

On the effect of duplication externalities, we have that

$$
\frac{\partial \bar{s}_{R}}{\partial \gamma}=\left.\frac{\partial \bar{s}_{R}}{\partial \gamma}\right|_{\bar{u}_{Y} / \bar{u}_{n}=\mathrm{constant}}+\frac{\partial \bar{s}_{R}}{\partial\left(\bar{u}_{Y} / \bar{u}_{n}\right)} \frac{\partial\left(\bar{u}_{Y} / \bar{u}_{n}\right)}{\partial \gamma}
$$

If $0 \leq \phi<1$, Eq. (83) entails that $\partial \bar{s}_{R} / \partial\left(\bar{u}_{Y} / \bar{u}_{n}\right) \leq 0$ and, therefore, $\partial \bar{s}_{R} / \partial \gamma>0$. If $\phi<0$, after simplification, we get that

$$
\frac{\partial \bar{s}_{R}}{\partial \gamma}=(1-\phi) \frac{\bar{g}_{H}\left(\xi-\bar{g}_{H}\right) \gamma^{2}+\alpha(1-\beta-\eta)\left[\left(\xi-(1-\gamma) \bar{g}_{H}\right)^{2}-\left(\xi-\bar{g}_{H}\right)^{2} i s \phi\right]}{\alpha \gamma^{2}(1-\beta-\eta)\left[\left(\xi-\bar{g}_{H}\right)(1-\phi)+\gamma \bar{g}_{H}\right]^{2}}>0,
$$

which completes the proof.

Therefore, according with intuition, an increase in R\&D spillovers (an increase of $\phi$ ) implies an increase in the long-run subsidy rate to $R \& D$, whereas and increase in duplication externalities (a decrease of $\gamma$ ) implies a decrease in its long-run rate. 


\section{Conclusion}

This paper devises a fiscal policy capable of making the decentralized economy to replicate the first-best optimum in an endogenous growth model with physical capital, human capital and R\&D. There are three sources of inefficiency in the model: monopolistic competition in the intermediategoods sector, externalities associated to the duplication of research effort, and spillovers in R\&D. The optimal growth path can be attained as a market equilibrium by instituting a subsidy to production of intermediate goods at a constant rate, which addresses the inefficiency caused by the presence of monopolistic competition, combined with a time-varying subsidy (or tax) to R\&D, which addresses the duplication externalities and spillovers in R\&D associated to the innovation process. Unlike much of the related literature, we also analyze the local stability of the efficient steady state, and a sufficient condition for saddle-path stability is derived. Furthermore, it is shown that the time path of the R\&D subsidy could be non-monotonic.

\section{Acknowledgements}

Manuel Gómez gratefully acknowledges financial support from the Spanish Ministry of Economy and Competitiveness through Grant ECO2011-25490. Tiago Sequeira gratefully acknowledges financial support from PTDC/EGE-ECO 102238/2008 - FCT (Fundação para a Ciência e Tecnologia).

\section{Appendix A. The model with no duplication externalities}

Up to now we have assumed that $\gamma<1$, when the dynamics of the economy can be described by the dynamic system (59)-(63). However, in the model without of duplication externalities, $\gamma=1$, Eq. (61) would not be well-defined, which shows that some differences arise in this case.

Let us now derive the dynamics system that drives the socially planned economy without duplication externalities, $\gamma=1$. Equating (37) and (41), using (44) to eliminate $g_{g_{n}}$ and (36) to eliminate $g_{\mu}$, we get that

$$
u_{Y}=\frac{\alpha(1-\beta-\eta) \xi}{(1-\alpha) \eta \delta \psi} .
$$

Therefore, $g_{u_{Y}}=-g_{\psi}=(1-\phi) g_{n}-g_{H}$, which combined with (63) yields

$$
g_{n}=\frac{\alpha[\beta \chi-(1-\eta) \xi]}{(1-\alpha) \eta-(1-\phi) \alpha \beta} .
$$

Hence, the optimal growth path is described in terms of the variables $r, \chi$ and $\psi$ by the third-order dynamic system (59), (60) and (55), where $u_{Y}$ and $g_{n}$ should be replaced with (A.1) and (A.2), respectively; i.e.,

$$
\begin{aligned}
g_{r} & =\frac{1-\beta-\eta}{\beta}(\xi-r)+\frac{(1-\alpha) \eta}{\beta[(1-\alpha) \eta-(1-\phi) \alpha \beta]}[\beta \chi-(1-\eta) \xi], \\
g_{\chi} & =\left(\frac{1}{\theta}-\frac{1-\eta}{\beta}\right) r+\chi-\frac{\rho}{\theta}, \\
g_{\psi} & =\xi\left[1-\frac{\alpha(1-\beta-\eta) \xi}{(1-\alpha) \eta \delta \psi}\right]-\left[\frac{\xi}{\delta \psi}+1-\phi\right] \frac{\alpha[\beta \chi-(1-\eta) \xi]}{(1-\alpha) \eta-(1-\phi) \alpha \beta} .
\end{aligned}
$$


It can be observed that the proof of Proposition 1 remains valid, so that it also yields the optimal steady state in the model with no duplication externalities, $\gamma=1$. The following proposition shows that the steady state is unstable in this case.

Proposition 5. In the absence of duplication externalities, $\gamma=1$, the steady state of the socially planned economy is unstable.

Proof. Note that in this case the interest rate $r$, as well as the knowledge-ideas ratio $\psi=H n^{\phi-1}$, is a predetermined variable as it can be expressed as

$$
r(0)=\beta \eta^{\eta /(1-\eta)} n(0)^{(1-\alpha) \eta /[\alpha(1-\eta)]}\left[u_{Y}(0) H(0) / K(0)\right]^{(1-\beta-\eta) /(1-\eta)},
$$

which, using that $u_{Y}(0)=\alpha(1-\beta-\eta) \xi /[(1-\alpha) \eta \delta \psi(0)]$ from (A.1), is uniquely determined by the initial values of the state variables $K, H$ and $n$. Therefore, saddle-path stability requires two stable roots.

Linearizing (A.3), (A.4) and (A.5) around its steady state yields

$$
\left(\begin{array}{c}
\dot{r} \\
\dot{\chi} \\
\dot{\psi}
\end{array}\right)=\left(\begin{array}{ccc}
J_{11} & J_{12} & 0 \\
J_{21} & J_{22} & 0 \\
0 & \bullet & J_{33}
\end{array}\right)\left(\begin{array}{c}
r-\bar{r} \\
\chi-\bar{\chi} \\
\psi-\bar{\psi}
\end{array}\right)=J \cdot\left(\begin{array}{c}
r-\bar{r} \\
\chi-\bar{\chi} \\
\psi-\bar{\psi}
\end{array}\right),
$$

where a dot replaces an element that is irrelevant for the analysis, and

$$
\begin{array}{ll}
J_{11}=-\frac{1-\beta-\eta}{\beta} \bar{r}, & J_{12}=\frac{(1-\alpha) \eta}{\eta(1-\alpha)-\alpha \beta(1-\phi)} \bar{r} \\
J_{21}=\left(\frac{1}{\theta}-\frac{1-\eta}{\beta}\right) \bar{\chi}, & J_{22}=\bar{\chi}, \\
J_{33}=\xi-(1-\phi) \bar{g}_{n}=\xi-\bar{g}_{H}>0 . &
\end{array}
$$

Let $\bar{J}$ denote the upper left $2 \times 2$ submatrix of $J$. The structure of $J$ entails that its eigenvalues are the two eigenvalues of $\bar{J}$, and the third eigenvalue is its last diagonal element, which is positive as $\xi-\bar{g}_{H}>0$ if (74) holds. Saddle-point stability can be assured then if the two eigenvalues of $\bar{J}$ have negative real parts, which holds if and only if the determinant of $\bar{J}$ is positive and its trace is negative. The trace of $\bar{J}$ can be obtained after simplification as $\operatorname{tr}(\bar{J})=\xi-\bar{g}_{H}>0$. Hence, the number of stable roots is less than the number of the predetermined variables and, therefore, it is not possible to make the system stable for arbitrary initial values of the predetermined variables; i.e., the steady state of the socially planned economy is instable.

\section{References}

Alvarez-Pelaez, M. J. and Groth, C. (2005). Too little or too much R\&D? European Economic Review, 49(2):437-456.

Arnold, L. G. (1998). Growth, welfare, and trade in an integrated model of human-capital accumulation and research. Journal of Macroeconomics, 20(1):81-105.

Arnold, L. G. (2000a). Endogenous growth with physical capital, human capital and product variety: A comment. European Economic Review, 44(8):1599-1605. 
Arnold, L. G. (2000b). Endogenous technological change: A note on stability. Economic Theory, 16(1):219-226.

Arnold, L. G. (2006). The dynamics of the Jones R\&D growth model. Review of Economic Dynamics, 9(1):143-152.

Barro, R. J. and Sala-i-Martin, X. (1995). Economic Growth. McGraw-Hill.

Benhabib, J., Meng, Q., and Nishimura, K. (2000). Indeterminacy under constant returns to scale in multisector economies. Econometrica, 68(6):1541-1548.

Benhabib, J. and Perli, R. (1994). Uniqueness and indeterminacy: On the dynamics of endogenous growth. Journal of Economic Theory, 63(1):113-142.

Brito, P. and Dixon, H. (2009). Entry and the accumulation of capital: A two state variable extension to the ramsey model. International Journal of Economic Theory, 5(4):333-357.

del Barrio-Castro, T., Lopez-Bazo, E., and Serrano-Domingo, G. (2002). New evidence on international R\&D spillovers, human capital and productivity in the OECD. Economics Letters, $77(1): 41-45$.

Eicher, T. S. and Turnovsky, S. J. (1999). Non-scale models of economic growth. Economic Journal, 109(457):394-415.

Engelbrecht, H.-J. (1997). International R\&D spillovers, human capital and productivity in OECD economies: An empirical investigation. European Economic Review, 41(8):1479-1488.

Funke, M. and Strulik, H. (2000). On endogenous growth with physical capital, human capital and product variety. European Economic Review, 44(3):491-515.

Gómez, M. A. (2005). Transitional dynamics in an endogenous growth model with physical capital, human capital and R\&D. Studies in Nonlinear Dynamics 85 Econometrics, 9(1):Article 5.

Gómez, M. A. (2011a). Duplication externalities in an endogenous growth model with physical capital, human capital, and R\&D. Economic Modelling, 28(1-2):181-187.

Gómez, M. A. (2011b). Stages of economic development in an innovation-education growth model. Studies in Nonlinear Dynamics 85 Econometrics, 15(4):Article 6.

Griliches, Z. (1992). The search for R\&D spillovers. Scandinavian Journal of Economics, 94(0):S2947.

Grossmann, V., Steger, T., and Trimborn, T. (2010). Dynamically optimal R\&D subsidization. CESifo Working Paper Series 3153, CESifo Group Munich.

Iacopetta, M. (2010). Phases of economic development and the transitional dynamics of an innovation-education growth model. European Economic Review, 54(2):317-330.

Iacopetta, M. (2011). Formal education and public knowledge. Journal of Economic Dynamics and Control, 35(5):676-693.

Jones, C. I. (1995). R\&D-based models of economic growth. Journal of Political Economy, 103(4):759-784. 
Jones, C. I. (2005). Growth and ideas. In Aghion, P. and Durlauf, S., editors, Handbook of Economic Growth, volume 1 of Handbook of Economic Growth, chapter 16, pages 1063-1111. Elsevier.

Jones, C. I. and Williams, J. C. (2000). Too much of a good thing? The economics of investment in R\&D. Journal of Economic Growth, 5(1):65-85.

Kortum, S. (1993). Equilibrium R\&D and the patent-R\&D ratio: U. S. evidence. American Economic Review, 83(2):450-457.

Lambson, V. E. and Phillips, K. L. (2007). Market structure and Schumpeterian growth. Journal of Economic Behavior \&3 Organization, 62(1):47-62.

Martínez-García, M. P. (2003). The general instability of balanced paths in endogenous growth models: the role of transversality conditions. Journal of Economic Dynamics and Control, 27(4):599 618.

Mino, K. (2001). Indeterminacy and endogenous growth with social constant returns. Journal of Economic Theory, 97(1):203-222.

Pessoa, A. (2005). 'Ideas' driven growth: the OECD evidence. Portuguese Economic Journal, $4(1): 46-67$.

Porter, M. E. and Stern, S. (2000). Measuring the 'ideas' production function: Evidence from international patent output. NBER Working Papers 7891, National Bureau of Economic Research, Inc.

Romer, P. M. (1990). Endogenous technological change. Journal of Political Economy, 98(5):S71S102.

Sequeira, T. N. (2011). R\&D spillovers in an endogenous growth model with physical capital, human capital, and varieties. Macroeconomic Dynamics, 15(2):223-239.

Steger, T. M. (2005). Welfare implications of non-scale R\&D-based growth models. Scandinavian Journal of Economics, 107(4):737-757.

Stokey, N. L. (1995). R\&D and economic growth. Review of Economic Studies, 62(3):469-89.

Strulik, H. (2007). Too much of a good thing? The quantitative economics of R\&D-driven growth revisited. Scandinavian Journal of Economics, 109(2):369-386. 